\title{
Impact of Multiple Adjustable Speed Drive System to Power System Harmonics
}

\author{
Taufik* $^{*}$, Erin Matsumoto* ${ }^{* *}$, and Makbul Anwari*** \\ *California Polytechnic State University, San Luis Obispo, USA. Email: taufik@calpoly.edu \\ ${ }^{* *}$ Chevron Energy Solutions, San Francisco, USA. Email: erin.matsumoto@gmail.com \\ ** Universiti Teknologi Malaysia, Skudai, Johor, Malaysia. Email: emakbul@ieee.org
}

\begin{abstract}
This paper presents harmonic analysis in a multiple adjustable speed drive system. In a system where multiple drives are fed from the same bus, harmonic currents produced by the various drives can reflect back to the power source and to the other drives causing distortion of the voltage waveform. If these harmonic currents are significant, they can potentially cause problems for the given system. Therefore studying the harmonics at various points in the system and comparing the values of total harmonic distortion can provide guidance in choosing appropriate filtering solutions. A two ac drive system was setup in a laboratory environment and the total harmonic distortion of the voltage and current was measured at several points within the system to observe how the harmonics interact with each other. The frequency of the drives and the torque of the motors were also varied to achieve a variable torque relationship to model heating, ventilation, and air condition loads.
\end{abstract}

Keywords - adjustable speed drives, power quality, harmonics.

\section{INTRODUCTION}

With the development and advancement of solid state adjustable speed drive technology, variable speed systems are becoming increasingly common in industrial and commercial applications. Adjustable speed drives (ASD), also known as variable speed drives or variable frequency drives (VFD), offer the benefit of energy efficiency for motor driven systems. With motors consuming more than 57 percent of all electric power generated and 20 percent of all energy used in the United States [1], these energy savings are significant, especially as demand for electricity increases. However, there are some drawbacks to VFD systems, mainly the harmonics created by the power electronic components in the drive. Harmonics are created by the switching scheme of the power electronic circuit inside the drive and can cause damage to power equipment on the utility side and/or sensitive loads on the customer side. Because of this, the Institute of Electrical and Electronic Engineers (IEEE) has developed standards for the amount of harmonic distortion a customer's load can inject back into the utility lines. To conform to this relatively new established guideline, it is a common practice nowadays for VFD systems to have line filters installed before them to reduce distortion caused by the VFDs back to the line. Doing so will therefore maintain a degree of power quality at the distribution level. More importantly, these line filters will also prevent the harmonic distortion to propagate back and further up at the transmission level.

This paper examines the total harmonic distortion at different locations in a multiple motor-driven system. One common application for variable frequency drives is for variable torque loads, often used in heating and ventilation systems. The main goal of the experiment conducted in this project is to simulate this variable torque loads under different loading conditions. With multiple motors operating from one VFD and multiple VFD's in the system, the harmonics created from the various loads could crosstalk and could further positively or negatively affect the magnitude of the distortion. However, by far, little has been known about the harmonic interaction between these drives on a single point of common coupling. Therefore, experimental results from this project will be important to further understand the interaction and hence will reveal how the drives interact with and affect each other. Moreover, results of the experiment will give insight to necessity of using line reactors and to identify the most appropriate locations to place line reactor filters to sufficiently mitigate the harmonics produced by the drives.

\section{EXPERIMENTAL MODEL \& PROCEDURES}

A variable speed driven system using [2] with variable torque loading was modelled in a laboratory environment. The system source was $240 \mathrm{VL}-\mathrm{L}, 3 \varphi$ power patched from a panel in the laboratory and represents a utility power source. This line was fed through a 2:1 ratio DeltaWye grounded transformer. The transformer configuration $(\Delta-Y)$ was selected because it is the most common for distribution systems. The 30 degree phase shift from delta to wye helps to rebalance any voltages that may be unbalanced. The grounded neutral on the wye side is recommended for safe drive operation and for the safety of those who may work on or around the equipment [2]. The secondary output of the transformer supplied power to two 2-hp adjustable speed drives. Each drive powered two 1-hp induction motors coupled with a dynamometer. The dynamometer was used to vary the load on the motor. The system setup is shown in Figure 1. 


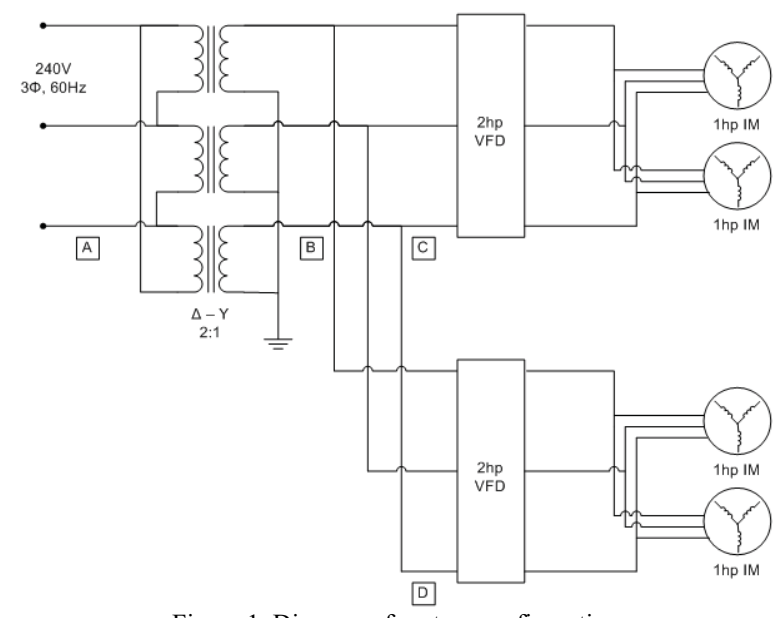

Figure 1. Diagram of system configuration

Since this experiment looked specifically at variable torque loads, it required the motor torque to remain proportional to the square of the motor speed at every loading profile [3]. This was achieved by appropriately adjusting the frequency of the VFD and the torque of the dynamometer so that the torque speed relationship held true. Figure 2 shows the torque-speed relationship tested and the drive frequency associated with each speed.

Motor Speed-T orque $\mathbf{C}$ haracteristics

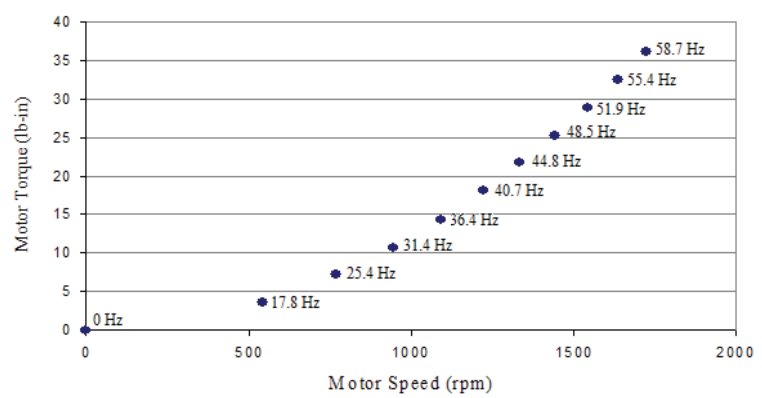

Figure 2. Torque and Frequency Values Associated with Measurements

Total harmonic distortions for current and voltage were measured at four different points within the system. One location is on the delta (primary) side of the transformer, the point of common coupling where ownership shifts from the utility to the customer. This is the point that utilities are concerned about harmonic distortion. Other measurements were made on the wye (secondary) side of the transformer and before each of the drives. Information obtained from these points can be used to find the most cost efficient way to place line reactors.

\section{LAB SETUP \& RESULTS}

This section outlines the procedure for recording measurements and reviews the necessary information to understanding the graphs and analysis that follow later in the paper. As previously discussed, one adjustable speed drive, labeled VFD \#1, was held at a constant frequency/speed and the two motors, M1 and M2, fed from that drive were held at an equal, constant torque. The frequency of the second drive, labeled VFD \#2, and motor loads, M3 and M4, were then varied to achieve 0 $100 \%$ of rated torque in $10 \%$ increments. Each time this procedure was completed for a specific speed/torque combination on the first drive, the data was compiled into graphs to observe the differences of harmonic distortion based on location and percentage of the rated load. Figure 3 is a simplified diagram of the system indicating the labeling convention of the data and graphs. Figure 4 is a picture of the actual lab setup showing the corresponding points of measurements as indicated in Figure 3.

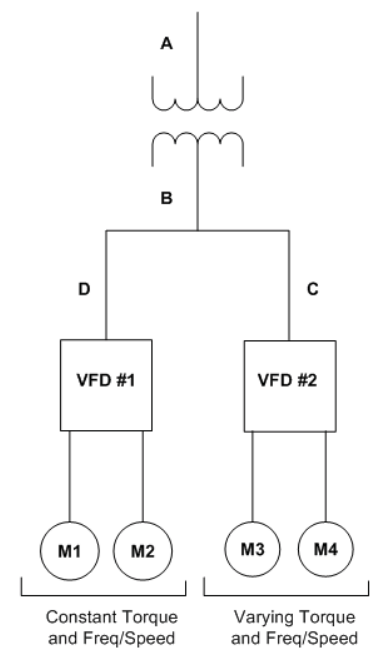

Figure 3. Simplified diagram of system configuration

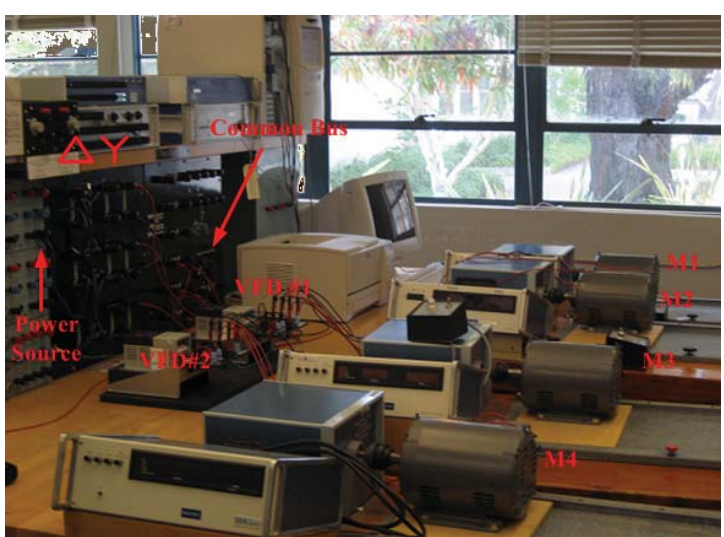

Figure 4. Snapshot of laboratory setup

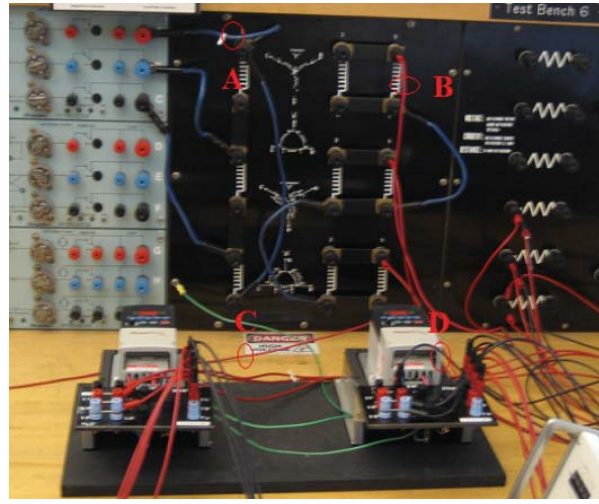

Figure 5. Adjustable speed drives in the laboratory setup 


\section{A. Analysis of Voltage Distortion}

Two examples of measured total harmonic distortion of the voltage were shown in Figures 6 and 7. The graphs illustrate how harmonic frequencies interact at locations in the system as the load on VFD \#2 increases. For all locations and loading conditions of $\mathrm{M} 1 / \mathrm{M} 2$, the total harmonic distortion of the voltage increased as the load on M3/M4 increased. However, some locations were affected more by changes in the motors loads with locations B, C, and D increasing at relatively the same rate which was faster than location $\mathrm{A}$. This makes sense since the transformer after point A functions as harmonic filter that blocks harmonic energy to creep back to the ac system. In addition to increasing at a similar pace, locations $\mathrm{B}, \mathrm{C}$, and $\mathrm{D}$ had $\mathrm{THD}_{\mathrm{V}}$ measurements that are close in value. The point within the system with the highest $\mathrm{THD}_{\mathrm{V}}$ can not be predicted because the variations at $\mathrm{B}, \mathrm{C}$, and $\mathrm{D}$ have no discernible pattern. In fact, the $\mathrm{THD}_{\mathrm{V}}$ should theoretically be the equal at B, C, and D because they are essentially at the point given that the resistance of the leads connecting the transformer to the drives is negligible. Location A, in comparison, increases at a steadier rate as it appears to be less affected by disturbances or inconsistencies in measurements.

Another observation from Figures 6 and 7 is that the difference in THD from point A to the rest of the points is between $0.5 \%$ to about $3 \%$. The lowest difference occurred when all motors are at $0 \%$ load. The largest difference is seen when all motors are fully loaded. These results are in agreement with the fact that at no load, line currents should be practically zero and voltage at the secondary side of the transformer (point B) should be just a pure reflection of the voltage at the primary side (point A). Since there line currents theoretically can not exist at no load conditions, voltages at points $\mathrm{B}, \mathrm{C}, \mathrm{D}$ must be equal. On the other hand, when significant line current is drawn by the drives, its harmonics energy will be the largest. The non-dominant line current harmonics which are pretty much negligible at low load become considerable to a certain extent at larger line currents since these non-dominant harmonics grow as larger current is being drawn by the drives. The larger dominant and non-dominant harmonics are translated by way of impedance to larger THD contents of the line voltages.

Another important characteristic of this system to note is that the voltage distortion measurements met IEEE 519-1992 standards for all conditions tested. This point corresponds to location $\mathrm{A}$ and the highest $\mathrm{THD}_{\mathrm{V}}$ measured there was $2.48 \%$ at M1/M2 at $50 \%$ and M3/M4 at $100 \%$. The other condition for meeting the recommended limits is each individual odd harmonic be less than $3 \%$. The individual voltage distortion for variable frequency drive systems is associated with the 5 th harmonic. The individual 5th harmonic content corresponding to the $\mathrm{THD}_{\mathrm{V}}$ measurement just mentioned was recorded with a magnitude of $3.8 \mathrm{~V}$. The voltage of the fundamental voltage was $235.7 \mathrm{~V}$ giving a ratio of 0.0161 or $1.61 \%$ of distortion.
Figures 8 to 11 view trends in voltage distortion with regards to M1/M2 load percentages at a specific location. In general, for measurements at $\mathrm{B}, \mathrm{C}$, and $\mathrm{D}$ lower percentages of the rated load of M1/M2 correspond to a lower percentages of voltage distortion. Although there is a correlation between the motor loads and the harmonic distortion at those points in the system, the experimental results reveal that this is not strictly followed. For example, at location B when M3/M4 are loaded at 20\%, the $\mathrm{M} 1 / \mathrm{M} 2$ at $0 \%$ measured a higher $\mathrm{THD}_{\mathrm{V}}$ than the M1/M2 10\% and 20\% settings. This trend can be seen at location A, but it does not follow the pattern as closely and more anomalies such as the example above are present. With the exception of location A, M1/M2 at $100 \%$ loading overall yields the highest $\mathrm{THD}_{\mathrm{V}}$ among other M1/M2 loadings. The data obtained at location A show that the higher THDs were measured at M1/M2 loading of $70 \%$ and $80 \%$. Theoretically, M1/M2 100\% loading should be producing the worst case THD due to the larger line current drawn by the drives. This anomaly may be contributed from external noise such as other equipment running in other rooms but connected to the same PCC as do these drives. Also due to limitations in the availability of laboratory equipment, the dynamometers used were not always the same. The rate at which the voltage distortion increases as the M3/M4 load increases appears to be independent of M1/M2 loading conditions.

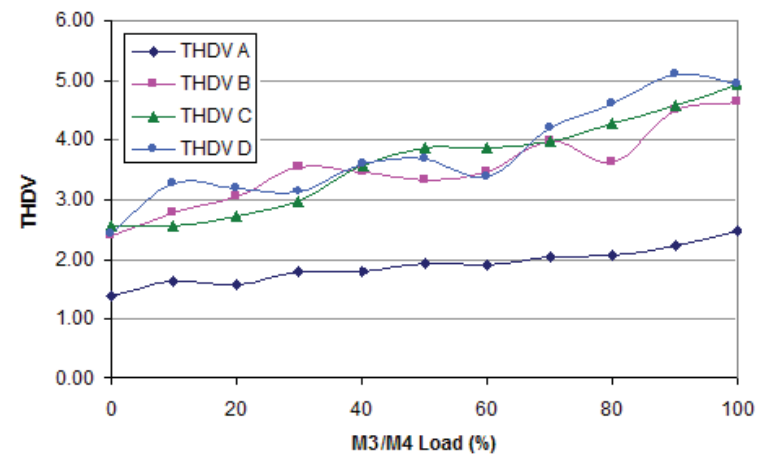

Figure 6. $\mathrm{THD}_{\mathrm{V}}$ vs. M3/M4 Load for M1/M2 at $50 \%$ of Rated Torque

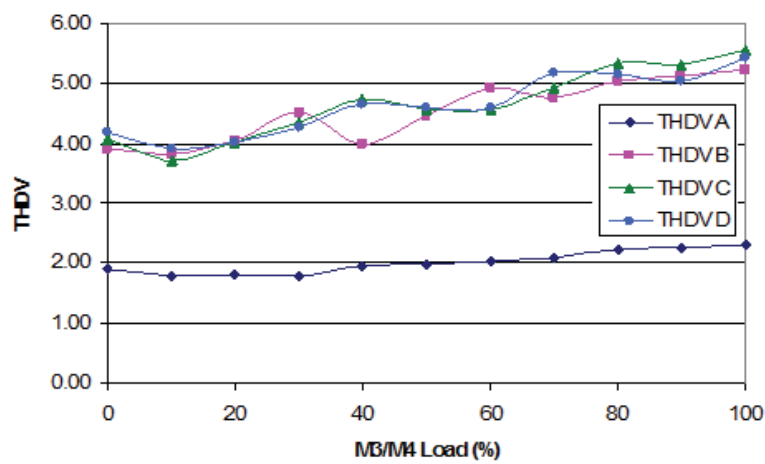

Figure 7. $\mathrm{THD}_{\mathrm{V}}$ vs. M3/M4 Load for M1/M2 at $100 \%$ of Rated Torque 


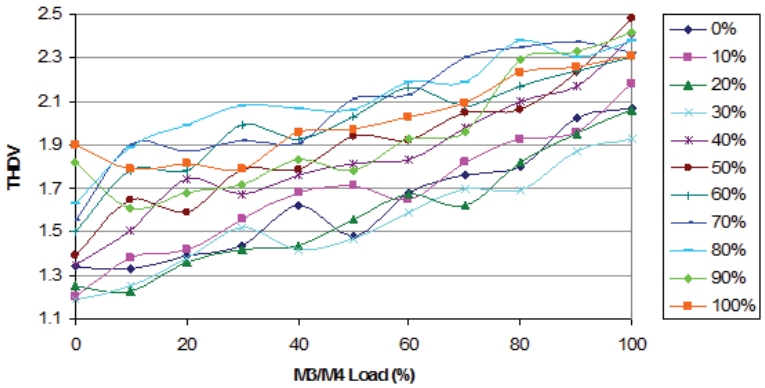

Figure 8. THD $\mathrm{V}_{\mathrm{V}}$ vs. M3/M4 Load at Location A

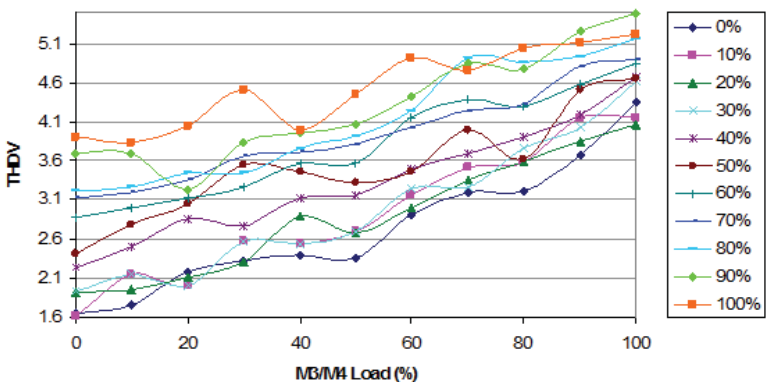

Figure 9. $\mathrm{THD}_{\mathrm{V}}$ vs. M3/M4 Load at Location B

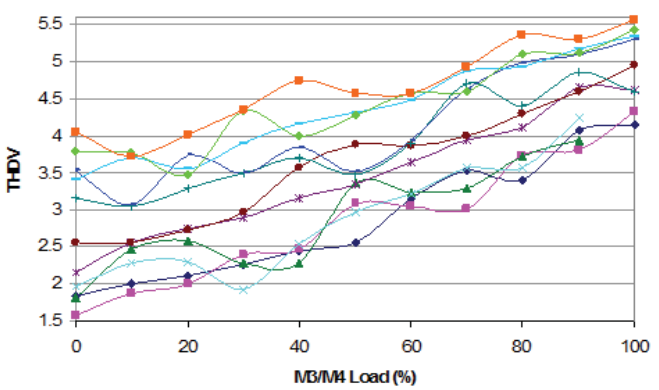

Figure 10. THD $v$ vs. M3/M4 Load at Location C

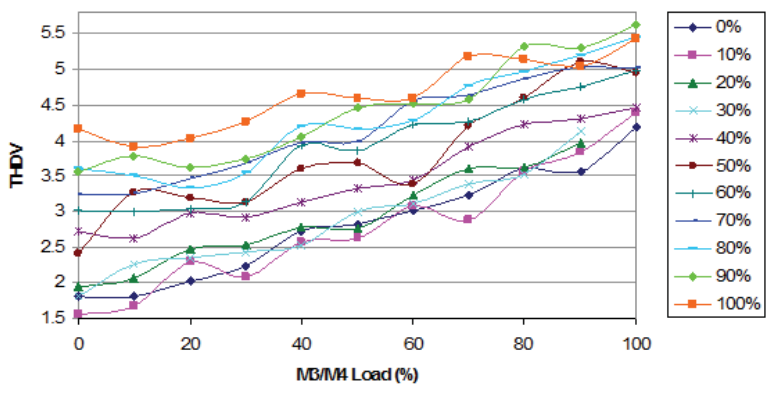

Figure 11. THD $\mathrm{V}_{\mathrm{V}}$ vs. M3/M4 Load at Location D

\section{B. Analysis of Current Distortion}

The analysis of current distortion is important, especially since the power quality issues relating to harmonic distortion of the current waveform are the responsibility of the customer compared to voltage distortion in which much of the responsibility of maintaining a clean voltage waveform lies on the utility [4]. For analysis of the current plots, the trends dealing with the specific location within the system will be presented.

The location plots (Figures 12 - 15) reveal that harmonic distortion is generally lower at higher loads.
This evident in the graphs because both an increase along the M3/M4 load axis and the data sets associated with higher loads for M1/M2 produce total current harmonic distortion (THDI) measurements that are smaller in value. Furthermore, it is interesting to observe that the $100 \%$ loading of M1/M2, with the exception at location C, yields lower THDs. Even at location C, the $100 \%$ loading of M1/M2 produced THDs that are in the lower range. This brings two implications. First and foremost is that the worst case $\mathrm{THD}_{\mathrm{I}}$ does not occur at high load, which may be counter-intuitive, but rather at the lower load as previously mentioned. Additional examination of the magnitude of the harmonic components reveals that this occurs because the fundamental component of the current increases at faster rate than the harmonic components. This means filter rating and selection for this multi-drive multi-motor connections should be done based on the lower loading values. Secondly, the lower THDs at higher loads preliminary show that certain cancellations of harmonic current from $\mathrm{C}$ and $\mathrm{D}$ at point $\mathrm{B}$. This is quite evident from the plot of current distortion at location B.

In contrast, the $\mathrm{THD}_{\mathrm{I}}$ of lightly loaded conditions for $\mathrm{M} 1 / \mathrm{M} 2$ at location $\mathrm{D}$ and the $\mathrm{THD}_{\mathrm{I}}$ of low percentage loads of M3/M4 at location $\mathrm{C}$ indicate that harmonic distortion is not only higher, but also less predictable for when the system is for low loads at those locations. Location B also shows high, erratic behavior with light loads, but to less extent than $\mathrm{C}$ and $\mathrm{D}$. Interestingly, location A displays contradictory behavior to this trend and yields lower $\mathrm{THD}_{\mathrm{I}}$ in this situation. Locations $\mathrm{A}$ and $\mathrm{B}$ have opposing characteristics when the motors are run at a small fraction of their rated values with the only electrical difference between the two locations is that harmonics are flow through the delta-wye transformer. On inspection of the individual harmonics contributions at these locations, location B has even-ordered harmonics that are filtered out and not reflected to the primary side of the transformer. Location A only follows the contrasting pattern of increasing loading corresponding to increasing $\mathrm{THD}_{\mathrm{I}}$ when $\mathrm{M} 1 / \mathrm{M} 2$ and $\mathrm{M} 3 / \mathrm{M} 4$ are in the range of $0 \%$ to $40 \%$, after which the THDI begins to decrease. The data for $50 \%$ loading of M1/M2 shows steady $\mathrm{THD}_{\mathrm{I}}$ is maintained before decreasing and the rest of the higher M1/M2 curves show decreasing pattern through the entire set of variations of M3/M4.

Measurements at location D show that when M1/M2 are at $0 \%$ load, the THD current at location D is highest. Recall that location D is where M1/M2 motors are located. Hence the THD current measured at location D while both motors M1 and M2 are not loaded should mostly be contributed from motors M3/M4. This is why the curve for $0 \% \mathrm{M} 1 / \mathrm{M} 2$ loading at location $\mathrm{C}$ is totally different from that of $0 \% \mathrm{M} 1 / \mathrm{M} 2$ loading at location $\mathrm{D}$. The data measured at location $\mathrm{C}$ at $0 \% \mathrm{M} 1 / \mathrm{M} 2$ loading should not be affected by M1/M2 (since they are at zero current anyways) at all but rather should be impacted by how much loadings exist at motors M3/M4.

Comparing the overall trends at each location, the distortion changes at location $\mathrm{D}$, with exception to the $0 \%$ 
and $10 \%$ data sets, were less drastic than at the other locations. This occurred because current measured at D remained fairly constant. Also, as expected the distortion is best at location $\mathrm{A}$.

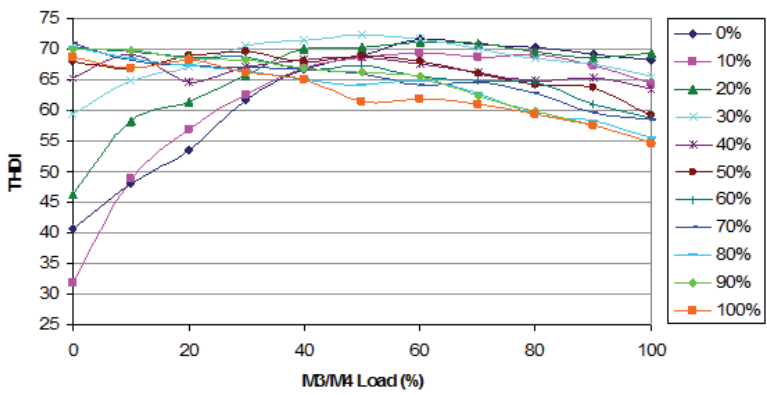

Figure 12. THD I vs. M3/M4 Load at Location A

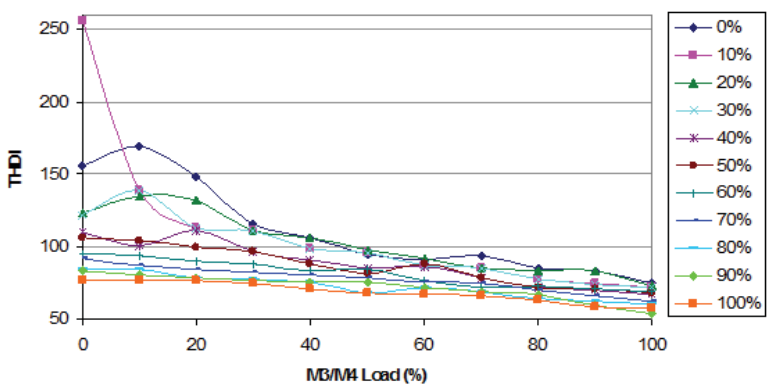

Figure 13. THD Is. M3/M4 Load at Location B

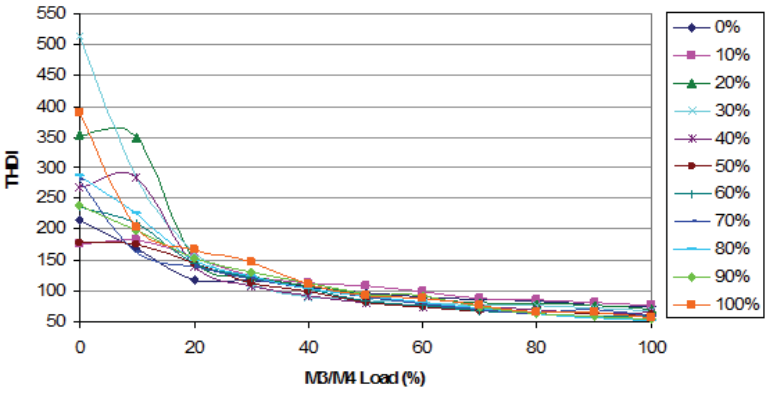

Figure 14. THD I vs. M3/M4 Load at Location C

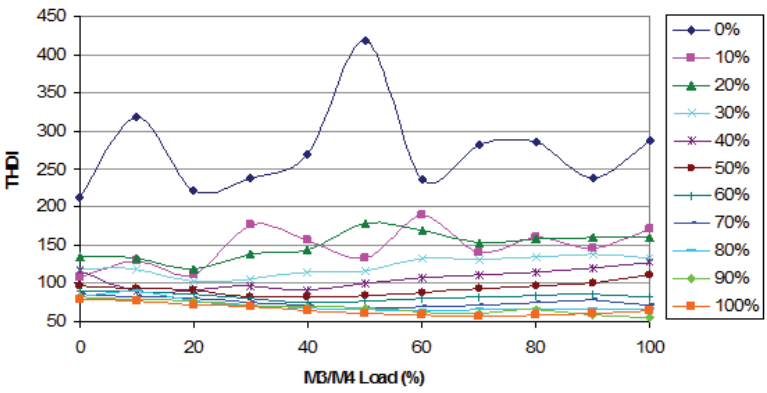

Figure 15. THD I vs. M3/M4 Load at Location D

\section{CONCLUSIONS}

The analysis of the data showed trends in the behavior of harmonics in the two drive system tested and can be used to determine the necessity and/or effectiveness of line reactors in a system with similar components and setup.
The total harmonic distortion of the input line voltage waveform was shown to have a sufficient degree of power quality with respect to the IEEE 519-1992 standards. At larger loads there were higher $\mathrm{THD}_{\mathrm{V}}$ measurements because there was more current being drawn, but this was not large enough to warrant filtering for this purpose. However, if this system did not have a transformer then the point of common coupling would be at the bus feeding the drives. The delta-wye configuration of the transformer reduces harmonics of line currents and thereby reducing harmonics of line voltages. Without the use of the transformer a line reactor should be used to maintain the power quality.

The total harmonic distortion of the line current revealed more interesting and useful information. The total harmonic distortion is lower at higher current while the total demand distortion increased with increasing loads. In other words, as the load increases the amount of harmonic distortion decreases relative to the fundamental component, but increases relative to the maximum demand of the load.

The harmonic current analysis also showed how the harmonics produced by each drive interact with each other and how they feed back into the line. The crosstalk between the drives is typically greater the further away the two motor torques and drive frequencies were from each other because the phase angle difference between the harmonic components is larger. However in some cases there appeared to be slight cancellations of the harmonics yielding lower THD values than when the two loads were equal. When the harmonic currents are flowing back to the source they are adding in value, but the total current distortions of each drive do not add in value. Additionally, the line feeding to the bus where the drives are powered has a smaller $\mathrm{THD}_{\mathrm{I}}$ than at the point before the drive supplying the smaller load.

\section{ACKNOWLEDGMENT}

We wish to acknowledge Rockwell Automation Allen Bradley for their generous donation of the adjustable speed drives to the Power Electronics lab at Cal Poly State University in San Luis Obispo, California.

\section{REFERENCES}

[1]. A. Bhaduri, "The Use of Variable Speed Drives in Existing HVAC Installations," Air Conditioning and Refrigeration Journal, July 2001.

[2]. Power Flex 4 Adjustable Frequency AC Drive User Manual, Allen-Bradley, Milwaukee, WI: Rockwell Automation, 2003.

[3]. M. F. McGranaghan, and D. R. Mueller, "Designing Harmonic Filters for Adjustable Speed Drives to Comply with IEEE-519 Harmonic Limits," IEEE Transactions on Industrial Applications, Vol. 35, pp. 312-318, 1999.

[4]. 1C.4.1 - Harmonic Distortion, PacfiCorp Utah Power, Engineering Standards and Technical Support Department, 1998. 